\title{
Pan-genome analysis of coronaviruses derived from major of canine and feline
}

\author{
Hai Ha Pham Thi ${ }^{1}$ and Thanh Luan Nguyen ${ }^{2}$ \\ ${ }^{1}$ Nguyen Tat Thanh University \\ ${ }^{2}$ Ho Chi Minh City University of Technology
}

November 19, 2020

\begin{abstract}
Coronaviruses (CoVs) are a well-known cause of severe enteric, respiratory, and systemic disease in a wide range of animals and in humans. To understand the route of disease origin and viral transmission in companion animals, a comparative pangenomic analysis of coronavirus sequences originating from major felines and canines were conducted. The average nucleotide identity (ANI) is a rapid procedure for assessing the very close antigenic relationship between feline CoV (FCoVs) and canine $\mathrm{CoV}(\mathrm{CCoVs})$ and ANI-based phylogenetic tree that clustered CoVs according to their respective host species. While pangenomic analysis demarcated strains clearly. The distribution of the clinical isolates all across the categories in the hierarchical phylogenetic model enabled the visualization of their original ecological niche rather than their isolation source, as infections are extremely rare events and evolutionary dead-ends. In polymorphism analysis, we found seven accessory gene clusters common to the $\mathrm{FCoV} / \mathrm{CCoV}$ category clade, including pantropic strains, that perform functions supporting their pathogenicity. In addition, the gene presence/absence among $\mathrm{FCoVs}$ and $\mathrm{CCoVs}$ would provide very valuable information on species-specific control measures against $\mathrm{CoV}$ disease, such as the selection of good markers for differentiating new species from common and/or pantropic isolates. Also, the virulent FCoV strains were grouped with human CoV strains NL63 and $229 \mathrm{E}$ confirming hypotheses stating that cats are highly susceptible to HCoVs, while dogs have low susceptibility to the virus. In conclusion, the combined analysis allows for better phylogenetic resolution and the implication of virus origins, recombination, and virus-host interaction, as well as biomarkers.
\end{abstract}

\section{Hosted file}

Pan-genome analysis of coronaviruses derived from major of canine and feline.pdf available at https://authorea.com/users/377248/articles/493972-pan-genome-analysis-ofcoronaviruses-derived-from-major-of-canine-and-feline

Hosted file

Figures.pptx available at https://authorea.com/users/377248/articles/493972-pan-genomeanalysis-of-coronaviruses-derived-from-major-of-canine-and-feline 